\title{
Properties of the young gas giant planet $\beta$ Pictoris b
}

\author{
Mickaël Bonnefoy ${ }^{1}$, Anthony Boccaletti ${ }^{2}$, Anne-Marie Lagrange ${ }^{3}$, \\ France Allard ${ }^{4}$, Christoph Mordasini ${ }^{1}$, Hervé Beust ${ }^{3}$, Gaël Chauvin ${ }^{3}$, \\ Julien H. V. Girard ${ }^{5}$, Derek Homeier ${ }^{4}$, Daniel Apai ${ }^{6,7}$, \\ Sylvestre Lacour ${ }^{2}$, Daniel Rouan ${ }^{2}$, Julien Rameau ${ }^{3}$ and \\ Hubert Klahr ${ }^{1}$ \\ ${ }^{1}$ Max Planck Institute for Astronomy, Königstuhl 17, D-69117 Heidelberg, Germany \\ email: bonnef oy@mpia-hd.mpg.de \\ ${ }^{2}$ LESIA, Observatoire de Paris, CNRS, University Pierre et Marie Curie Paris 6 and \\ University Denis Diderot Paris 7, 5 place Jules Janssen, 92195 Meudon, France \\ ${ }^{3}$ UJF-Grenoble 1 / CNRS-INSU, Institut de Planétologie et d'Astrophysique de Grenoble \\ (IPAG) UMR 5274, Grenoble, F-38041, France \\ ${ }^{4}$ CRAL, UMR 5574, CNRS, Université de Lyon, École Normale Supérieure de Lyon, 46 Allée \\ d'Italie, F-69364 Lyon Cedex 07, France \\ ${ }^{5}$ European Southern Observatory, Casilla 19001, Santiago 19, Chile \\ ${ }^{6}$ Department of Astronomy, 933 N. Cherrt Avenue, Tuscon, AZ 85721, USA \\ ${ }^{7}$ Department of Planetary Sciences, The University of Arizona, 1929 E. University Blvd., \\ Tuscon, AZ 85721, USA
}

\begin{abstract}
The young $\left(12_{-4}^{+8} \mathrm{Myr}\right)$ and nearby $(19.44 \pm 0.05 \mathrm{pc})$ star $\beta$ Pictoris is considered one of the best laboratories for the study of early phases of planetary systems formation since the identification of an extended debris disk surrounding the star in 1984. In 2009, we imaged at $3.8 \mu \mathrm{m}$ with $\mathrm{NaCo}$ at VLT a gas giant planet around $\beta$ Pictoris, roughly along the disk mid-plane, with a semi-major axis between 8 and $14 \mathrm{AU}$. We present here the first images of the planet in the $\mathrm{J}(1.265 \mu \mathrm{m}), \mathrm{H}(1.66 \mu \mathrm{m})$, and M' $(4.78 \mu \mathrm{m})$ bands obtained between 2011 and 2012. We used these data to build the 1-5 $\mu \mathrm{m}$ spectral energy distribution (SED) of the companion, and to consolidate previous semi-major axis (8-10 AU) estimates. We compared the SED to seven atmospheric models to derive $\mathrm{T}_{\text {eff }}=1700 \pm 100 \mathrm{~K}$. We used the temperature and the luminosity of $\beta$ Pictoris $\mathrm{b}$ to estimate new masses for the companion. We compared these masses to independent constraints set by the orbital parameters and the radial velocities and use them to discuss the formation history of the object.
\end{abstract}

Keywords. instrumentation: adaptive optics, high angular resolution, stars: $\beta$ Pictoris, Exoplanets: formation, evolution, atmosphere

\section{Introduction}

$\beta$ Pictoris is a nearby $(19.44 \pm 0.05 \mathrm{pc})$ intermediate-mass star $(\mathrm{A} 6 \mathrm{~V})$, member of the $\beta$ Pictoris moving group $\left(12_{-4}^{+8} \mathrm{Myr}\right)$. It is known to bear a structured debris disk seen nearly edge-on (Smith \& Terrile 1984). In 2010, we announced the direct detection at L' band $(3.8 \mu \mathrm{m})$ of a $7-11 \mathrm{M}_{\mathrm{Jup}}$ planet orbiting the star, roughly along the dust disk position angle (PA), with a semi-major axis (s.m.a.) between 8 and 14 AU (Lagrange et al. 2010). $\beta$ Pictoris b is the closest planet (physically) to its star ever imaged. The discovery further confirmed that the system remains one of the best testbeds for planet formation theories and for the study of planet/disk interactions.

Nevertheless, our knowledge of the physical characteristics of the planet (mass, $T_{e f f}$ ), like those of imaged planets or companions, is mostly based on predictions of 

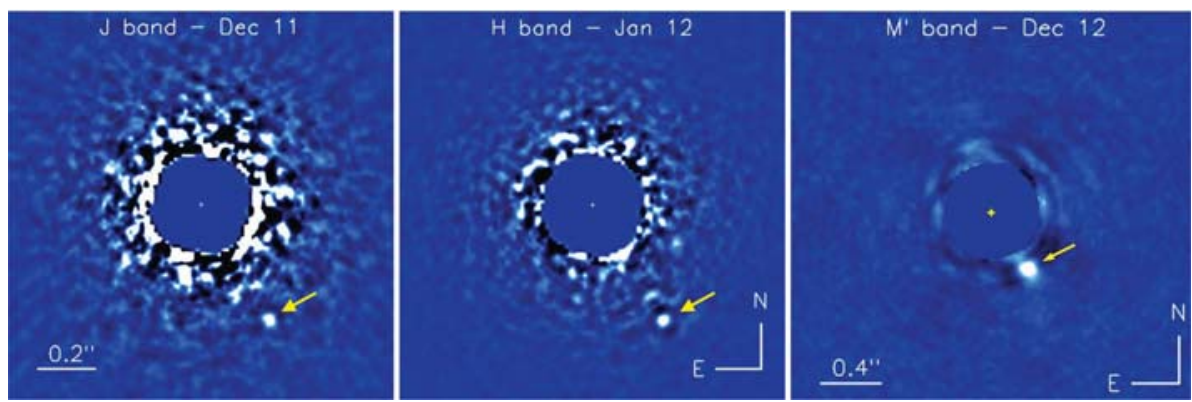

Figure 1. Re-detection of $\beta$ Pictoris b (arrow) in the J (left), $\mathrm{H}$ (middle), and M' bands.

"hot-start" evolutionary models. These models assume that the objects start their evolution following a spherical collapse from an arbitrary large initial radius (Chabrier et al. 2000, Baraffe et al. 2003). Alternative "cold-start" models were proposed in order to predict the properties of planets produced by accretion of gas. They hypothesize that the accreted gas loses all of its gravitational energy through a super-critical accretion shock. They predict giant planets with low initial entropies, e.g. cooler and dimmer objects than those predicted by the "hot start" models for the same mass.

We made new observations in order to derive accurate estimates of the physical (mass, $T_{\text {eff }}, \log \mathrm{g}$ ) and chemical (composition, chemistry) properties of $\beta$ Pictoris b. We used these estimates to discuss predictions of evolutionary models and to sketch a possible scenario for the planet.

\section{New observations of $\beta$ Pictoris $\mathbf{b}$}

We used NaCo at VLT to obtain four new high-contrast observations of $\beta$ Pictoris in the $\mathrm{J}(1.226 \mu \mathrm{m}), \mathrm{H}(1.65 \mu \mathrm{m})$, and M' $(4.78 \mu \mathrm{m})$ between fall-2011 and 2012 . The so-called "pupil-stabilized" mode was used and enabled for angular differential imaging of the star (Marois et al. 2006). Data were analyzed using two independent data reduction pipelines. Each pipeline used four different algorithms, including the most recent developments in the field (Lafrenière et al. 2007, Soummer et al. 2012). The planet is detected in each band (see Fig. 1). We derive $\mathrm{J}=14.0 \pm 0.3 \mathrm{mag}, \mathrm{H}=13.5 \pm 0.2 \mathrm{mag}$, and $\mathrm{M}^{\prime}=11.0 \pm 0.3$ mag.

\section{Planet properties}

\subsection{Astrometry}

The position of $\beta$ Pictoris $\mathrm{b}$ with respect to the star was extracted from the H-band data. It pursues the monitoring of the orbital motion of the planet (Chauvin et al. 2012, Bonnefoy et al. 2013, Lagrange et al. in prep). We applied Markov-Chain Monte Carlo simulations on the current set of astrometric data points, including new astrometry from unpublished NaCo observations (Lagrange et al., in prep). These simulations constrain the s.m.a of $\beta$ Pictoris b to 8-10 AU with a maximum of realizations for a s.m.a of 9 AU. For s.m.a. of 9 and 10 AU, radial-velocity measurements (Lagrange et al. 2012) place upper limits of 12 and $15.5 \mathrm{M}_{\mathrm{Jup}}$ on the dynamical mass of the planet, respectively. This is the first time that such constraints are placed on the mass of a directly imaged planet. We also confirm from this analysis that the orbit has a low eccentricity $(e \leqslant 0.17)$. 


\subsection{Colors, luminosity, and effective temperature}

We compared the new photometry at J, H, and M' band, together with previous photometry obtained in the $\mathrm{K}_{\mathrm{s}}$ band (Bonnefoy et al. 2011), to that of young objects and mature field MLT dwarfs in color-color and color-magnitude diagrams. Colors based on the $\mathrm{J}, \mathrm{H}$, and $\mathrm{K}_{\mathrm{s}}$ magnitudes are comparable to those of early-L (L0-L4) field dwarfs and to those of young early L-type companions. We used this crude constraint on the spectral type of the planet and bolometric corrections valid for young $\mathrm{L}$ dwarfs (Todorov et al. 2010, Faherty et al. 2013$)$ to derive a luminosity estimate $\log _{10}\left(\mathrm{~L} / \mathrm{L}_{\odot}\right)=-3.87 \pm 0.08$. Colors based on the M' band photometry appear redder than those of field L dwarfs.

We combined the present set of photometric data points (Lagrange et al. 2009, Quanz et al. 2010, Bonnefoy et al. 2011, 2013) to build the 1-5 $\mu \mathrm{m}$ spectral energy distribution (SED) of the planet. The SED was compared to synthetic fluxes generated from a set of seven atmospheric models. These models account for the formation of dust grains in the photosphere of the object. But they consider either an extreme (AMESCond, BT-Cond), partial (BT-Settl 2010, BT-Settl 2012, DRIFT-PHOENIX), or a lack of (Dusty) gravitational settling of the dust following its formation. Models with photospheric dust tend to better reproduce the observations (see Fig. 2). All models converge toward $\mathrm{T}_{\text {eff }}=1700 \pm 100 \mathrm{~K}$. They do not provide a firm estimate of the surface gravity and metallicity of the object.

\subsection{Mass and initial entropy}

We used the constraints on the effective temperature and luminosity of the planet as input of evolutionary models to derive new mass estimate. "hot-start" models predict masses from 7 to $13 \mathrm{M}_{\mathrm{Jup}}$ for the planet, that match constrains on its dynamical mass (Section 3.1). On the contrary, predictions from "cold-start" models of (Marley et al. 2007) (MA07) and (Spiegel \& Burrows 2012) (SB12), which are limited to planets with masses in the range 1-10 $\mathrm{M}_{\mathrm{Jup}}$, do not reproduce the observed properties of the planet.

We therefore considered the more recent "warm-start" evolutionary models of SB12. These models account for intermediate initial conditions to those considered for the "coldstart" and "hot-start" cases. These initial conditions reflect different possible formation pathways and are parameterized by a choice of initial entropy $\left(\mathrm{S}_{\text {init }}\right)$. Predicted $\mathrm{T}_{\text {eff }}$ and luminosities increase with the mass and $S_{\text {init }}$. As a consequence, the corresponding properties of (Section 3.2) $\beta$ Pictoris b can only be reproduced for a restrained set of masses $\left(M \geqslant 6 M_{J u p}\right)$ and $S_{\text {init }}$. $S_{\text {init }}$ can be further constrained to $S_{\text {init }} \geqslant 9.8 \mathrm{k}_{\mathrm{B}} /$ baryon using the dynamical mass of $\beta$ Pictoris b for a s.m.a of 9 AU (see Fig 2). This lower limit corresponds to $\mathrm{S}_{\text {init }}$ values intermediate between those corresponding to the "cold-start" and "hot-start" models of MA07.

\section{The origin of $\beta$ Pictoris $b$}

The initial entropy can not directly be used to discuss the origins of the planet. But it could be compared to similar constraints brought on other directly imaged planets (e.g. Marleau \& Cumming 2013). Nevertheless, the narrow range of possible s.m.a., the lower limit on the mass of the planet inferred from "warm-start" models, and the upper limit on the dynamical mass can be used as inputs of formation models. Formation models of H. Klahr (Klahr et al in prep.) predict that massive clumps $\left(M \geqslant 3 M_{J u p}\right)$ can only be formed in the gravitationally unstable disk surrounding a star as massive as $\beta$ Pictoris at more than 45 AU (Rameau et al. 2013). Therefore, according to these models, $\beta$ Pictoris $\mathrm{b}$ would have migrated inward significantly without accreting too much disk material. Conversely, planets as massive as $\beta$ Pictoris $\mathrm{b}$ are formed in population synthesis models 

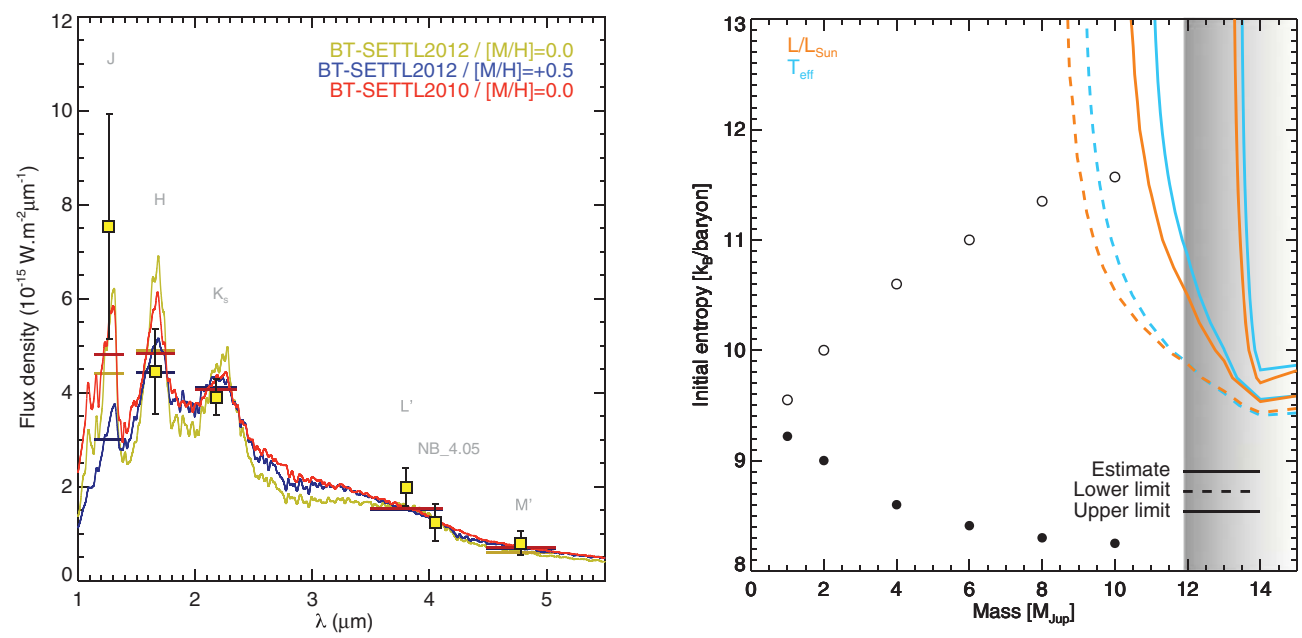

Figure 2. Left: Spectral energy distribution of $\beta$ Pictoris b (squares) compared to best-matching synthetic fluxes predicted by BT-Settl atmospheric models. Right: Predictions of "warm-start" evolutionary models for different planet masses and initial entropies $\left(S_{\text {init }}\right)$ compared to $\beta$ Pictoris b properties for an age of 8 to $20 \mathrm{Myr}$. Models reproduce the observed temperature and bolometric luminosity of the planet for a restrained range of mass and initial entropies (delimited by the dashed and dashed-dotted lines). Independent upper-limits on the mass of the planet exclude part of this parameter space (shaded area) and enable to constrain the initial entropy of the planet to values higher than $9.8 \mathrm{k}_{\mathrm{B}}$ /baryon (according to these "warm-start" models). This lower limit on the initial entropy of the planet fall in-between initial entropies corresponding to "cold-start" (black points), and "hot-start" (open circles) models.

based on the core-accretion scenario (including type I and type II migration) around 12 Myr old stars as massive as $\beta$ Pictoris (Mordasini et al. 2012). These planets are part of the envelope of the distribution of synthetic planets. They are not found beyond 15 AU.

\section{References}

Smith, B. A., \& Terrile, R. J. 1984, Science, 226, 1421

Lagrange, A.-M., Bonnefoy, M. et al. 2010, Science, 329, 57

Chabrier, G., Baraffe, I., Allard, F., \& Hauschildt, P. 2000, ApJ, 542, 464

Baraffe, I., Chabrier, G., Barman, T. S., Allard, F., \& Hauschildt, P. H. 2003, ApJ, 402, 701

Marois, C., Lafrenière, D., Macintosh, B., \& Doyon, R. 2006, ApJ, 647, 612

Lafrenière, D., Marois, C., Doyon, R., Nadeau, D., \& Artigau, E. 2007, ApJ, 660, 770

Soummer, R., Pueyo, L., \& Larkin, J. 2012, ApJ, 755, 28

Chauvin, G., Lagrange, A.-M., Beust, H., Bonnefoy, M., et al. 2012, A\&A A, 542, 41

Bonnefoy, M., Boccaletti, A., Lagrange, A.-M., et al. 2013, A $\mathscr{E} A, 555,107$

Lagrange, A.-M., De Bondt, K., Meunier, N., Sterzik, M., et al. 2012, A\& $A$, 542, 18

Bonnefoy, M., Lagrange, A.-M., Boccaletti, A., Chauvin, G., et al. 2011, A\&A A, 528, 15

Todorov, K., Luhman, K. L., \& McLeod, K. K. 2010, ApJ, 714, 84

Faherty, J. K., Rice, E. L., \& Cruz, K. L. 2013, AJ, 145, 2

Lagrange, A.-M., Gratadour, D., Chauvin, et al. 2009, A\&A A, 493, 21

Quanz, S. P., Meyer, M. R., Kenworthy, M. A., et al. 2010, ApJ, 722, 49

Marley, M. S., Fortney, J. J., Hubickyj, O., et al. 2007, ApJ, 655, 541

Spiegel, D. S. \& Burrows, A. 2012, ApJ, 745, 174

Marleau, G.-D., \& Cumming, A. 2013, MNRAS, Submitted. astro-ph: 1302.1517

Rameau, J., Chauvin, G., Lagrange, A.-M., \& Klahr, H. 2013, A\&A, 553, 60

Mordasini, C., Alibert, Y., Klahr, H., \& Henning, T. 2012, A\& A, 547, 111 


\section{Discussion}

TRIAUD: What is the probability that the planet will transit, and when will it be transiting?

Bonnefoy: The orbital fits are still compatible with the "transit event" of 1981. As far as I remember, the predictions for the next transit are around 2019. But I am not sure that we can give a probability at this time. The orbital parameters are not known with a sufficient accuracy yet.

MERU: Just a quick comment about disk instability. We were looking at migration of planets and gravitationally unstable disks, and we do actually find that they migrate pretty rapidly. I don't know whether it is too rapid for this, or not? But it is certainly a possibility.

Bonnefoy: Yes exactly. There are also the papers of Zhu (et al. 2012), Michael (et al. 2011), and there have been a lot of work actually claiming that the timescales are $\sim 10^{3}$ $10^{4}$ years. That is very short. But I would be a bit cautious since we know from population synthesis models of Mordasini et al. (for instance) that the efficiency of migration is not very well known so far. So we have to be a bit careful I think.

CURrIE: So it is the first flux-calibrated detection at M'. I had the first detection at M'. Rome was not built in a day, and neither $\beta$ Pictoris b. So how do your conclusions about the luminosity, the initial entropy, and the mass change if you allow the planet to form in 3 to 5 million years.

BonNEFOY: So your question relates to your paper that you posted on astro-ph. This new paper is presenting a detection at $3.1 \mu \mathrm{m}$. Thayne (Currie) reanalysed data taken at other wavelengths we got, and that he got from Gemini. And your hypothesis is that the planet might have been formed a bit later compared to the age of the star. Well, it is a possibility of course. I like this picture. But you base your hypothesis on the estimation of the radius of the object that you derive fitting the actual SED with atmospheric models and using a dilution factor. Actually, I refrained from using this information to discuss anything about the age of the object because I believe that atmospheric models are not well calibrated right now to allow for such an analysis. It would be nice to use different models such as the one of Fortney (on top of Allard's and Helling's models used in Bonnefoy et al. 2013, and Madhusudhan's and Burrow's models used in Currie et al. 2013) and try to estimate a more reliable range of radius for the objet.

MARoIs: Do you have a clear detection indicating that $\beta$ Pic b is about to go back toward the star? Do you see it decelerating?

Bonnefoy: Actually, I don't know! We know it is probably close to the quadrature. It is at a very favorable position for spectroscopic monitoring. But I don't remember what are the other constraints that Anne-Marie (Lagrange) got from the very last astrometric data points. I advise you to take a look at her poster.

BILler: Two quick questions. First, your plot for the simulated planets of mass and separations. If you take the simulated planets, if you plot them into evolutionary models, how does that compare with $\beta$ Pic b itself? 
Bonnefoy: We tried to do that with the new code of Christoph Mordasini which couples the formation and the evolution of these planets. Christoph is making two hypothesis for the accretion shock: he is assuming that the shock can be fully efficient. In this case, you have a "cold-start". Or you can also have the opposite case, e.g. a "hot-start". And both the "cold-start" and the "hot-start" hypothesis are working for $\beta$ Pictoris! So you can form the planet with the observed luminosity and temperature. The idea is that beyond the issue of the efficiency of the accretion shock, there are other ingredients entering the game like the deuterium-burning, and some other energy sources like the infall of planetesimals in the enveloppe that can modify the cooling curve of the planet. That is an ongoing work.

BILLER: Second question. What are the prospects for getting high resolution spectra and do a similar analysis as Quinn Konopacky did for HR8799c?

BonNefoy: For $\beta$ Pictoris b, it is going to be a bit more difficult because the planet is at a slightly shorter separation and the contrast with the star remains high. We could first try to get a low resolution spectrum with SPHERE. It might give us some insights on the atmospheric composition of the planet. But there is no instrument in the South that could collect a high resolution spectrum. SINFONI at VLT for instance is the same kind of instrument as OSIRIS (at Keck, used by Konopacky et al.). But it is limited by persistence effects. So you can not really use it to do this kind of thing. 